International Journal of High Speed Electronics and Systems, Vol. 5, No. 4 (1994) 765-768

(C) World Scientific Publishing Company

\title{
AUTHOR INDEX \\ VOLUME 5 (1994)
}

Akagi, J., see Obara, M.

Almogy, G., see Scherer, A.

Andreadakis, N. C., see Soole, J. B. D.

Andreadakis, N. C., see Zah, C. E.

Aoki, M., Suzuki, M., Sano, H., Sasaki, S., Kawano, T., and

Kodera, H., Monolithic Integration of DFB Lasers and

Electroabsorption Modulators Using In-Plane Quantum

Energy Control of MQW Structures

Asbeck, P. M., see Wang, K.-C.

Asbeck, P. M., Chang, M. F., and Pedrotti, K., Future Directions for HBT Development

Bayraktaroglu, B. and Higgins, J. A., HBTs for Microwave Power Applications

Beam III, E., see Liu, W.

Bhat, R., see Soole, J. B. D.

Bhat, R., see Zah, C. E.

Bhat, R. J., see Scherer, A.

Bowers, J. E., see Nagarajan, R.

Caneau, C., see Soole, J. B. D.

Caneau, C., see Zah, C. E.

Chandrasekhar, S., The Progress and Performance of Long

Wavelength OEIC Photoreceivers Incorporating Heterojunction Bipolar Transistors

Chang, C., see Wang, K.-C.

Chang, C.-Y., see Wu, C.-C.

Chang-Hasnain, C. J., Wu, Y. A., Eng, L. E., and Li, G. S., Vertical Cavity Surface Emitting Laser Arrays for Wavelength Division Multiplexing Applications

Chang, M. F., see Asbeck, P. M.

Chen, Y. K., Humphrey, D. A., Fan, L., Hamm, R. A., Sivco, D., Cho, A. Y., and Tate, A., Noise Characteristics of InP-Based Heterostructure Bipolar Transistors

Cho, A. Y., see Chen, Y. K.

Choquette, K. D., see Schneider Jr., R. P.

Comfort, J., see Meyerson, B. S.

Corcoran, J., see Wang, K.-C.

Crabbe, E., see Meyerson, B. S.

Curtis, L., see Zah, C. E.
5 (1994) 253-274

5 (1994) 543-567

5 (1994) 111-133

5 (1994) 91-109

5 (1994) 67-90

5 (1994) 213-252

5 (1994) 493-527

5 (1994) 275-348

5 (1994) 411-471

5 (1994) 111-133

5 (1994) 91-109

5 (1994) 543-567

5 (1994) 1-44

5 (1994) 111-133

5 (1994) 91-109

5 (1994) 45-65

5 (1994) 213-252

5 (1994) 731-764

5 (1994) 569-592

5 (1994) 493-527

5 (1994) 395-410

5 (1994) $395-410$

5 (1994) 625-666

5 (1994) 473-491

5 (1994) 213-252

5 (1994) 473-491

5 (1994) 91-109 
Eng, L. E., see Chang-Hasnain, C. J.

5 (1994) 569-592

Fan, L., see Chen, Y. K.

Favire, F., see Zah, C. E.

Franzon, P. D., see Washabaugh, S.

Fu, C. C., see Yu, D. C. H.

Gabara, T., Pulsed Low Power CMOS

Gamelin, J., see Zah, C. E.

Hagerott Crawford, M., see Schneider Jr., R. P.

Hamm, R. A., see Chen, Y. K.

Harame, D. L., see Meyerson, B. S.

Higgins, J. A., see Bayraktaroglu, B.

Huang, K.-F., see Wu, C.-C.

Humphrey, D. A., see Chen, Y. K.

Iga, K., GaInAsP/InP Vertical Cavity Surface Emitting Lasers in 1.3 and $1.5 \mu \mathrm{m}$-Wavelength Band

Ishibashi, T., Yamauchi, Y., Sano, E., Nakajima, H., and Matsuoka, Y., Ballistic Collection Transistors and Their Applications

Jewell, J. L., see Scherer, A.

Kawano, T., see Aoki, M.

Khatibzadeh, A., see Liu, W.

Kim, T., see Liu, W.

Knudsen, K., see Wang, K.-C.

Kodera, H., see Aoki, M.

Kornblit, A., see Yu, D. C. H.

Koza, M. A., see Soole, J. B. D.

LeBlanc, H. P., see Soole, J. B. D.

Lee, K. H., see Yu, D. C. H.

Lee, T. P., see Zah, C. E.

Li, G. S., see Chang-Hasnain, C. J.

Lin, H. D., see Yu, D. C. H.

Lin, P. S. D., see Zah, C. E.

Liu, W., Beam III, E., Kim, T., and Khatibzadeh, A., Recent

Developments in GaInP/GaAs Heterojunction Bipolar Transistors

Lott, J. A., see Schneider Jr., R. P.

Mahoney, D. D., see Zah, C. E.

Matsuoka, Y., see Ishibashi, T.

5 (1994) 395-410

5 (1994) 91-109

5 (1994) 203-212

5 (1994) 135-143

5 (1994) 159-177

5 (1994) 91-109

5 (1994) 625-666

5 (1994) 395-410

5 (1994) 473-491

5 (1994) 275-348

5 (1994) 731-764

5 (1994) 395-410

5 (1994) 529-542

5 (1994) 349-379

5 (1994) 543-567

5 (1994) $67-90$

5 (1994) 411-471

5 (1994) 411-471

5 (1994) 213-252

5 (1994) 67-90

5 (1994) 135-143

5 (1994) 111-133

5 (1994) 111-133

5 (1994) 135-143

5 (1994) 91-109

5 (1994) 569-592

5 (1994) 135-143

5 (1994) 91-109

5 (1994) 411-471

5 (1994) 625-666

5 (1994) 91-109

5 (1994) 349-379

Meyerson, B. S., Harame, D. L., Stork, J., Crabbe, E., Comfort, J., and Patton, G., Silicon:Germanium Heterojunction Bipolar Transistors; From Experiment to Technology

Morgan, R. A., High-Performance, Producible Vertical-Cavity Lasers for Optical Interconnects

Nagarajan, R., Tauber, D., and Bowers J. E., High Speed Semiconductor Lasers

Nagle, H. T., see Washabaugh, S.

Nakajima, H., see Ishibashi, $\mathrm{T}$.

Nakwaski, W., see Osiński, M.

Nubling, R. B., see Wang, K.-C.

Obara, M. and Akagi, J., The Design and Fabrication of High-Speed HBT Circuits for Fiber-Optic Communication Systems

5 (1994) 473-491

5 (1994) 593-623

5 (1994) $1-44$

5 (1994) 203-212

5 (1994) 349-379

5 (1994) 667-730

5 (1994) $213-252$

5 (1994) 253-274 
O'Brien, J., see Scherer, A.

Osiński M. and Nakwaski, W., Thermal Effects in Vertical-Cavity

5 (1994) 543-567

Surface-Emitting Lasers

5 (1994) 667-730

5 (1994) 91-109

5 (1994) 473-491

Patton, G., see Meyerson, B. S.

5 (1994) $179-202$

5 (1994) 493-527

5 (1994) 213-252

5 (1994) 213-252

5 (1994) 145-157

5 (1994) 349-379

5 (1994) 67-90

5 (1994) 67-90

Sasaki, S., see Aoki, M.

Scherer, A., O'Brien, J., Almogy, G., Xu, W.-H., Yariv, A., Jewell, J. L., Uomi, K., Yoo, B. J., and Bhat, R. J., Vertical Cavity Surface Emitting Lasers with Dielectric Mirrors

5 (1994) 543-567

Schneider Jr., R. P., Lott, J. A., Hagerott Crawford, M., and Choquette, K. D., Epitaxial Design and Performance of AlGaInP Red (650-690 nm) VCSELs

Sheng, N.-H., see Wang, K.-C.

Sivco, D., see Chen, Y. K.

Soole, J. B. D., LeBlanc, H. P., Andreadakis, N. C., Bhat, R., Caneau, C., and Koza, M. A., Monolithic InP Reflection-Grating Multiplexer/Demultiplexers for WDM Components Operating in the Long Wavelength Fiber Band

Stork, J., see Meyerson, B. S.

Sugahara, H., GaAs HBT Reliability

Suzuki, M., see Aoki, M.

Tai, K.-C., see Wu, C.-C.

Tate, A., see Chen, Y. K.

Tauber, D., see Nagarajan, R.

Uomi, K., see Scherer, A.

Wang, K.-C., Nubling, R. B., Pedrotti, K., Sheng, N.-H., Asbeck, P. M., Poulton, K., Corcoran, J., Knudsen, K., Yuan, H.-T., and Chang, C., AlGaAs/GaAs HBTs for Analog and Digital Applications

Washabaugh, S., Franzon, P. D., and Nagle, H. T., SABSA: Switching-Activity-Based State Assignment

Wu, C.-C., Tai, K.-C., Huang, K.-F., and Chang, C.-Y., Reliability of Proton Implanted Vertical Cavity Surface Emitting Lasers

Wu, Y. A., see Chang-Hasnain, C. J.

$\mathrm{Xu}$, W.-H., see Scherer, A.

Yamauchi, Y., see Ishibashi, T.

Yan, R. H., see Yu, D. C. H.

Yariv, A., see Scherer, A.

Yoo, B. J., see Scherer, A.

Young, W. C., see Zah, C. E.

Yu, D. C. H., Lee, K. H., Kornblit, A., Fu, C. C., Yan, R. H., and Lin H. D., New Robust $\mathrm{n}^{+} / \mathrm{p}^{+}$Dual-Gate CMOS Technology Optimized for Low Power Operation

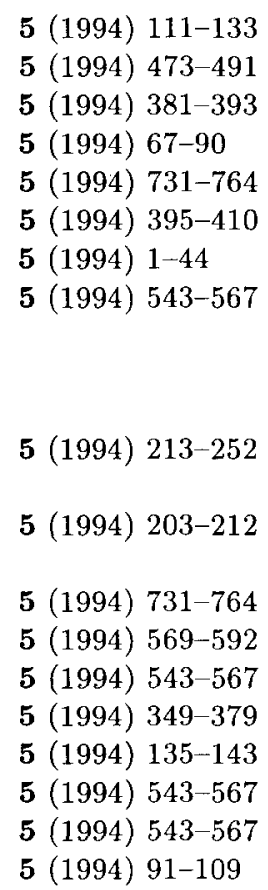

5 (1994) 213-252

5 (1994) 203-212

5 (1994) 731-764

5 (1994) 569-592

5 (1994) $543-567$

5 (1994) 349-379

5 (1994) 135-143

5 (1994) 543-567

5 (1994) 543-567

5 (1994) 91-109

5 (1994) 135-143 
Yuan, H.-T., see Wang, K.-C.

5 (1994) 213-252

Zah, C. E., Gamelin, J., Pathak, B., Favire, F., Lin, P. S. D., Andreadakis, N. C., Bhat, R., Caneau, C., Curtis, L.,

Mahoney, D. D., Young, W. C., and Lee, T. P., Multiwavelength

Light Source with Integrated DFB Laser Array and Star Coupler

for WDM Lightwave Communications

5 (1994) 91-109 